\title{
Fenología de las especies arbóreas de la Reserva Nacional Tambopata, Perú
}

\section{Phenology of tree species of the Tambopata National Reserve, Peru}

\author{
Gustavo A. Martínez-Sovero ${ }^{1}$, Consuelo Rojas-Idrogo ${ }^{1,2}$, Guillermo E. Delgado- \\ Paredes $^{1,2^{*}}$, Felipe Zuñe-Da Silva ${ }^{3}$, Alexander Huamán-Mera ${ }^{4}$, Yuriko S. Murillo- \\ Domen $^{5}$, Donald J. Brightsmith ${ }^{6}$
}

\begin{abstract}
The Amazonian ecosystem is one of the most important in the world and also one of the least studied, especially in regard to its vegetation and its phenophases. The objective of this study was to identify and describe the phenological patterns of the most frequent large tree species in the Tambopata National Reserve, Madre de Dios, Peru. The study was conducted between the years 2010 to 2017; by establishing five $50 \times 30 \mathrm{~m}$ plots for each forest type: Aguajal Forest, Bajío Forest, Successional Forest and Tierra Firme Forest. In each plot, all tree individuals with a diameter $\geq 10 \mathrm{~cm}$ were marked and identified at $1.30 \mathrm{~m}$ above the ground. An analysis of indicator species by habitat was performed, and the resulting taxa were periodically observed to evaluate the flower bud, flower, immature fruit and mature fruit phenophases. In addition, the influence of precipitation and temperature variables on their phenological responses was evaluated. A total of 1958 individuals were recorded, belonging to 57 families, 173 genera and 300 species; the Fabaceae, Moraceae and Annonaceae families stand out with the highest number of taxa and Arecaceae, with the highest number of individuals. Eight indicator species were analyzed, two for each forest type. Except in the Aguajal forest, the flower bud and mature fruit phenophases reached their maximum values in September and December-January. This information will contribute to a better understanding of the phenodynamics of each of the forest types in the Tambopata National Reserve.
\end{abstract}

Key words: Phenophase analysis, Amazon rainforest, floristic composition, indicator species, phenodynamics, Madre de Dios.

\section{Resumen}

El ecosistema amazónico es uno de los más importantes del mundo y también uno de los menos estudiados, en especial su vegetación y sus fenofases. El objetivo del presente trabajo fue identificar y describir los patrones fenológicos de las especies arbóreas de gran porte más frecuentes de la Reserva Nacional Tambopata, Madre de Dios, Perú. El estudio se realizó entre los años de 2010 a 2017; mediante el establecimiento de cinco parcelas de $50 \times 30 \mathrm{~m}$ por cada tipo de bosque: Bosque de Aguajal, Bosque de Bajío, Bosque Sucesional y Bosque de Tierra Firme. En cada parcela se marcaron e identificaron todos los individuos arbóreos con diámetro $\geq 10 \mathrm{~cm}$, a $1.30 \mathrm{~m}$ por encima del suelo. Se hizo un análisis de especies indicadoras por hábitats y los taxones resultantes se observaron periódicamente, para evaluar las fenofases de botón floral, flor, fruto inmaduro y fruto maduro. Además, se evaluó la influencia de las variables precipitación y temperatura sobre sus respuestas fenológicas. Se registraron 1958 individuos, pertenecientes a 57 familias, 173 géneros y 300 especies; destacan las familias Fabaceae, Moraceae y Annonaceae con el mayor número de taxa y Arecaceae con más individuos. Se analizaron ocho especies indicadoras, dos por cada tipo de bosque. Con excepción del Bosque de Aguajal, las fenofases botón floral y fruto maduro alcanzaron sus máximos valores en septiembre y diciembre-enero. Esta información contribuirá a un mejor entendimiento de la fenodinámica de cada uno de los tipos de bosques de la Reserva Nacional Tambopata.

Palabras clave: Análisis de fenofases, bosque amazónico, composición florística, especies indicadoras, fenodinámica, Madre de Dios.

Fecha de recepción/Reception date: 22 de diciembre de 2020

Fecha de aceptación/Acceptance date: 28 de mayo de

\footnotetext{
${ }^{1}$ Facultad de Ciencias Biológicas, Universidad Nacional Pedro Ruiz Gallo. Perú.

${ }^{2}$ Laboratorio General de Biotecnología, Vicerrectorado de Investigación, Universidad Nacional Pedro Ruiz Gallo. Perú.

${ }^{3}$ Programa de Pós-Graduação em Ciências Biológicas (Botânica), Universidade Federal do Rio de Janeiro. Brasil.

${ }^{4}$ Facultad de Ingeniería Forestal y Ambiental, Universidad Nacional de Jaén. Perú.

${ }^{5}$ Universidade Federal de Viçosa, Laboratório de Ecologia e Evolução de Plantas- LEEP. Brasil.

${ }^{6}$ Department of Veterinary Pathobiology, Texas A\&M University. USA.

*Autor para correspondencia; correo-e: guidelg2015@yahoo.es
} 


\section{Introducción}

En las últimas décadas el efecto del cambio climático, expresado en diversos tipos de estrés ambiental, ha recibido una creciente atención evidenciada en numerosos estudios recientes (Häder y Barnes, 2019). Los efectos del cambio climático en las plantas son dramáticos, debido a que estas carecen de movilidad. Los factores abióticos que más afectan la fenología de las plantas son: la temperatura (Körner y Basler, 2010), el fotoperiodo y la precipitación (Jackson, 2009); principalmente, en áreas tropicales y subtropicales (Pires et al., 2018). La fenología vegetal es un indicador integrativo ambiental del cambio climático, expresado en la permanencia, senescencia o abscisión de hojas, floración y maduración de frutos (Workie y Debella, 2018). Además, la fenología integra otros factores vitales como la duración, la magnitud y la sincronización de los ciclos de las plantas (Pires et al., 2018).

En las regiones tropicales, es relativamente accesible visualizar las floraciones, sobre todo en ambientes urbanos, de allí su aplicación en evaluaciones referentes al efecto del cambio climático (Du et al., 2015). Sin embargo, pocas son las investigaciones de comunidades arbóreas que podrían explicar las respuestas de las plantas ante el efecto del cambio climático (Davies et al., 2013). Los estudios fenológicos se han basado en observaciones de una sola especie como Ginkgo biloba L. (Matsumoto et al., 2003), o bien en taxones de un solo género como Protea (Daru et al., 2019) y otros de cobertura continental, por ejemplo, el realizado por Wolkovich et al. (2012).

En bosques amazónicos y, específicamente en el Perú, los trabajos fenológicos son escasos. Solamente se han realizado sobre: aspectos fenológicos estacionales en la Reserva Nacional Tambopata (Madre de Dios, Perú) (Girardin et al., 2016), los factores climáticos que determinaron la disminución de la vegetación en las sequías amazónicas de 2005 y 2010 (Zhao et al., 2017) y de las relaciones entre la insolación y la precipitación en la producción y caída de hojas (Wagner et al., 2017).

Por lo anterior, el presente trabajo tuvo como objetivos identificar y describir los patrones fenológicos de las especies arbóreas más frecuentes del bosque amazónico de la Reserva Nacional Tambopata. 


\section{Materiales y Métodos Ubicación y caracterización del área de estudio}

La investigación se realizó entre enero de 2010 y diciembre de 2017, en la Reserva Nacional Tambopata (RNT) del Centro de Investigaciones de Tambopata (Tambopata Research Center/TRC), ubicada a 250 msnm, en las coordenadas 433162 E - 8548037 N, cerca al límite del Parque Nacional Bahuaja Sonene, región Madre de Dios, Perú (Figura 1). El área de estudio abarca una superficie de 1613 ha, con una precipitación media anual de $2925 \mathrm{~mm}$ y una temperatura media anual de $25^{\circ} \mathrm{C}$.

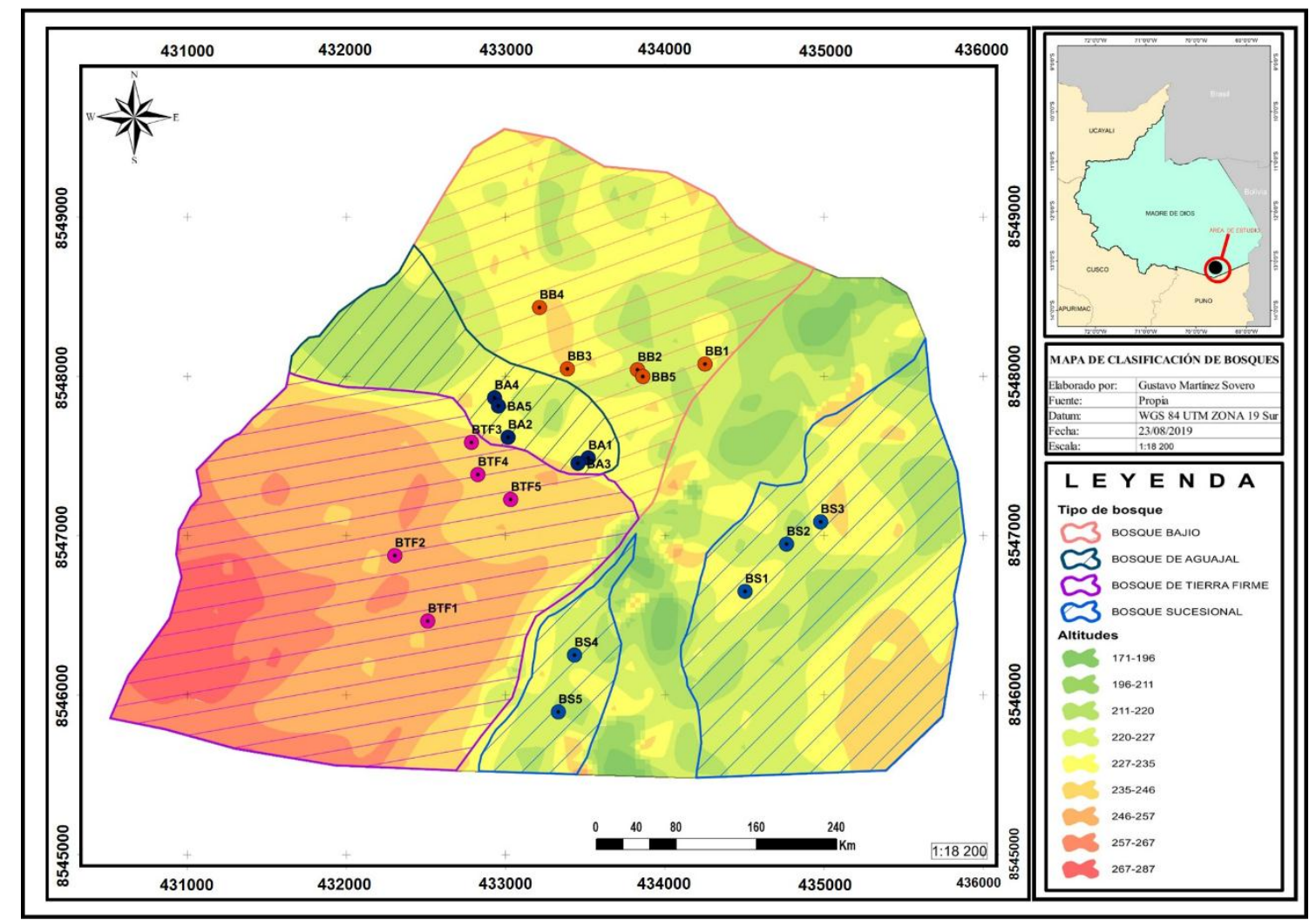

Figura 1. Ubicación del área de estudio y distribución de parcelas en la RNT, Madre de Dios, Perú.

Ribeiro et al. (1999) clasificó el área en cuatro tipos de bosques: Bosque de Aguajal (Ba), con suelos inundados durante casi todo el año y predominando Mauritia flexuosa L.f. (aguaje); Bosque de Bajío (Bb), con suelos ocasionalmente 
inundados y con pocas especies emergentes como Dipteryx micrantha Harms (shihuahuaco) y Ceiba pentandra (L.) Gaertn. (ceiba); Bosque Sucesional (Bs), situado en la llanura del río y dominado por taxones de rápido crecimiento como Erythrina poeppigiana (Walp.) O.F.Cook y E. ulei Harms, Triplaris americana L. (tangarana) y parches de Guadua sp. (paca); y Bosque de Tierra Firme (Bt), con suelos arcillosos, con especies emergentes como Hymenaea courbaril L. (azúcar huayo) y Eschweilera coriacea (DC.) S.A. Mori.

\section{Colecta de datos y procesamiento de muestras}

Las parcelas fueron instaladas siguiendo la orientación del sistema de trochas del Centro de Investigación. Se evaluaron 20 parcelas de $50 \times 30 \mathrm{~m}$, cinco para cada tipo de bosque, que representan 3.5 veces el área de $20 \times 20 \mathrm{~m}$ recomendada para una subparcela (Phillips et al., 2016). En cada parcela se registraron todos los individuos arbóreos con DAP (diámetro a $1.30 \mathrm{~m}$ del suelo) $\geq 10 \mathrm{~cm}$. Los individuos registrados $(n=1958)$ se colectaron, herborizaron e identificaron a partir de visitas virtuales a colecciones botánicas: Trópicos (https://www.tropicos.org/), The Plant List (http://www.theplantlist.org/) y GBIF (https://www.gbif.org/); Así como de consultas a especialistas y visitas a herbarios físicos próximos al área de estudio: Herbario Alwyn Gentry (HAG), herbario Vargas (CUZ) y herbario Selva Central de Oxapampa (HOXA).

Posteriormente, se ordenaron por familias de acuerdo al sistema propuesto por el grupo para la filogenia de Angiospermas (APG IV, 2016); los ejemplares se depositaron en el Herbario de la RNT. Enseguida, se procedió a identificar taxones indicadores por tipo de bosque, a partir de la fidelidad y afinidad de la especie por el tipo de hábitat (Dufrêne y Legendre, 1997), cuyos individuos se monitorearon mensualmente con binoculares para evaluar las siguientes fenofases: botón floral, flor, fruto inmaduro y fruto maduro. En la toma de datos fenológicos, se utilizó una modificación de la escala de Fournier (1974) para ausencia/presencia de fenofases, en la que 0 indica ausencia y 1 presencia. Los datos de precipitación y temperatura para el periodo de estudio se obtuvieron de 
la Estación Meteorológica de la RNT y la Estación Meteorológica de Puerto Maldonado (Madre de Dios).

\section{Análisis de datos}

Se construyeron tres matrices: una florística, una fenológica y una ambiental. A partir de la matriz florística, se llevó a cabo un análisis de ordenamiento NMDS (Non-metric Multidimensional Distance Scaling) (Kruskal, 1964) que utiliza la matriz de disimilitud entre parcelas, aplicando la distancia de Bray-Curtis. Además, se aplicó un análisis PERMANOVA (Permutational Multivariate Analysis of Variance) para verificar diferencias significativas entre los grupos formados y se usó el método de rarefacción para comparar la riqueza de especies entre tipos de bosques (hábitats); se estimó el máximo número de especies a partir del índice de Chao2, como medida de eficiencia del muestreo (Colwell, 2013). Para verificar las especies indicadoras de tipos de hábitats, se hizo un análisis ISA (Indicator Species Analysis) (Dufrêne y Legendre, 1997), que determina la significancia de las especies en análisis a partir de una prueba de Monte Carlo para probar la hipótesis nula de que las especies en estudio no tenían un valor indicador (IndVal).

Posteriormente, para verificar la influencia de las variables ambientales sobre las fenológicas, se aplicó un análisis CCA (Canonical Correlation Analysis) (McCune y Grace, 2002). Se obtuvo la relación de los promedios de precipitación y temperatura con los promedios de las fenofases para las especies indicadoras por tipo de bosque, para la media de cada mes entre los años de 2010-2017.Por último, se verificó la correlación de esos meses con las fenofases en estudio para las especies indicadoras. Se utilizó la prueba de Monte Carlo con 1000 permutaciones para determinar la significancia de la ordenación. Todos los análisis se efectuaron con el ambiente estadístico R 3.6.3 (R Core Team, 2021) y el programa Fitopac 2.1 (Shepherd, 2010). 


\section{Resultados Composición florística}

En los cuatro tipos de bosque se registraron 1958 individuos, agrupados en 57 familias, 173 géneros y 300 especies. Las familias con mayor número de géneros fueron Fabaceae (19), Euphorbiaceae (12), Malvaceae (12), Moraceae (12) y Annonaceae (11). Las familias con más especies fueron Fabaceae (36), Moraceae (27) y Annonaceae (18); y las que agruparon más individuos fueron Arecaceae (607), Euphorbiaceae (144), Fabaceae (142) y Myristicaceae (122) (Cuadro 1). Además se registraron 68 especies para el Bosque de Aguajal, 142 para el Bosque de Bajío, 82 para el Bosque Sucesional y 158 para el Bosque de Tierra Firme; cabe señalar que una especie se observó en más de un tipo de bosque.

Cuadro 1. Relación de familias y número de géneros, especies e individuos, evaluados en el Bosque de Aguajal (Ba), Bosque de Bajío (Bb), Bosque Sucesional (Bs) y Bosque de Tierra Firme (Bt) en la RNT, Madre de Dios, entre los años 2010-2017.

\begin{tabular}{lccccccc}
\hline Familias & $\begin{array}{c}\text { Géneros } \\
\text { Núm. }\end{array}$ & $\begin{array}{c}\text { Especies } \\
\text { Núm. }\end{array}$ & \multicolumn{2}{c}{ Bosques/Núm. individuos } & $\begin{array}{c}\text { Total } \\
\text { (indiv.) } \\
\text { Núm. }\end{array}$ \\
\hline Anacardiaceae & 3 & 4 & 0 & 4 & 8 & 1 & 13 \\
Annonaceae & 11 & 18 & 20 & 17 & 33 & 14 & 84 \\
Apocynaceae & 3 & 7 & 1 & 4 & 7 & 25 & 37 \\
Araliaceae & 2 & 2 & 0 & 2 & 2 & 7 & 11 \\
Arecaceae & 9 & 10 & 308 & 126 & 124 & 49 & 607 \\
Bignoniaceae & 3 & 4 & 1 & 0 & 3 & 6 & 10 \\
Boraginaceae & 1 & 2 & 0 & 1 & 1 & 4 & 6 \\
Burseraceae & 3 & 6 & 5 & 5 & 2 & 9 & 21 \\
Calophyllaceae & 1 & 1 & 6 & 0 & 0 & 0 & 6 \\
Cannabaceae & 2 & 2 & 0 & 7 & 0 & 0 & 7 \\
Capparaceae & 1 & 1 & 3 & 0 & 3 & 0 & 6 \\
Caricaceae & 1 & 1 & 0 & 4 & 0 & 0 & 4 \\
Celastraceae & 2 & 3 & 0 & 1 & 0 & 0 & 1 \\
Chrysobalanaceae & 2 & 6 & 6 & 3 & 0 & 5 & 14 \\
Clusiaceae & 2 & 2 & 2 & 0 & 0 & 1 & 3
\end{tabular}




\begin{tabular}{|c|c|c|c|c|c|c|c|}
\hline Combretaceae & 2 & 2 & 0 & 4 & 9 & 1 & 14 \\
\hline Ebenaceae & 1 & 1 & 0 & 0 & 0 & 1 & 1 \\
\hline Elaeocarpaceae & 1 & 4 & 2 & 4 & 2 & 5 & 13 \\
\hline Euphorbiaceae & 12 & 16 & 27 & 8 & 37 & 72 & 144 \\
\hline Fabaceae & 19 & 36 & 14 & 26 & 54 & 48 & 142 \\
\hline Icacinaceae & 1 & 1 & 0 & 1 & 0 & 0 & 1 \\
\hline Lacistemataceae & 1 & 1 & 0 & 1 & 0 & 0 & 1 \\
\hline Lamiaceae & 1 & 2 & 0 & 0 & 0 & 2 & 2 \\
\hline Lauraceae & 6 & 14 & 2 & 10 & 15 & 16 & 43 \\
\hline Lecythidaceae & 4 & 5 & 10 & 6 & 0 & 16 & 32 \\
\hline Linaceae & 1 & 1 & 2 & 0 & 0 & 1 & 3 \\
\hline Macgraviaceae & 1 & 1 & 0 & 0 & 0 & 1 & 1 \\
\hline Malpighiaceae & 1 & 1 & 1 & 0 & 0 & 0 & 1 \\
\hline Malvaceae & 12 & 14 & 40 & 27 & 11 & 34 & 112 \\
\hline Melastomataceae & 1 & 4 & 1 & 1 & 1 & 3 & 6 \\
\hline Meliaceae & 0 & 13 & 1 & 62 & 16 & 16 & 95 \\
\hline Moraceae & 12 & 27 & 7 & 41 & 31 & 27 & 106 \\
\hline Myristicaceae & 4 & 8 & 7 & 57 & 35 & 23 & 122 \\
\hline Myrtaceae & 3 & 5 & 0 & 1 & 0 & 7 & 8 \\
\hline Nyctaginaceae & 2 & 5 & 1 & 7 & 1 & 14 & 23 \\
\hline Ochnaceae & 1 & 1 & 0 & 2 & 0 & 0 & 2 \\
\hline Olacaceae & 3 & 3 & 0 & 1 & 0 & 6 & 7 \\
\hline Opiliaceae & 1 & 1 & 0 & 1 & 0 & 2 & 3 \\
\hline Phyllanthaceae & 2 & 2 & 2 & 1 & 0 & 0 & 3 \\
\hline Phytolacaceae & 1 & 1 & 0 & 2 & 0 & 0 & 2 \\
\hline Picramniaceae & 1 & 1 & 0 & 1 & 0 & 0 & 1 \\
\hline Piperaceae & 1 & 2 & 0 & 0 & 2 & 0 & 2 \\
\hline Polygonaceae & 2 & 2 & 0 & 2 & 34 & 2 & 38 \\
\hline Putranjivaraceae & 1 & 1 & 0 & 0 & 0 & 1 & 1 \\
\hline Rubiaceae & 7 & 7 & 0 & 2 & 11 & 3 & 16 \\
\hline Rutaceae & 2 & 2 & 0 & 1 & 0 & 7 & 8 \\
\hline Sabiaceae & 1 & 2 & 0 & 0 & 0 & 2 & 2 \\
\hline Salicaceae & 2 & 6 & 0 & 8 & 8 & 5 & 21 \\
\hline Sapindaceae & 2 & 4 & 0 & 5 & 5 & 0 & 10 \\
\hline Sapotaceae & 4 & 14 & 4 & 13 & 1 & 23 & 41 \\
\hline Simarubaceae & 1 & 1 & 1 & 0 & 0 & 2 & 3 \\
\hline Siparunaceae & 1 & 2 & 0 & 1 & 0 & 4 & 5 \\
\hline
\end{tabular}




\begin{tabular}{lccccccc} 
Staphylaceae & 1 & 1 & 0 & 1 & 0 & 0 & 1 \\
Trigonaceae & 1 & 1 & 0 & 1 & 0 & 0 & 1 \\
Urticaceae & 3 & 12 & 4 & 16 & 39 & 20 & 79 \\
Violaceae & 3 & 3 & 0 & 4 & 0 & 7 & 11 \\
Vochysiaceae & 1 & 1 & 0 & 0 & 0 & 1 & 1 \\
\hline Total & 173 & 300 & 478 & 492 & 495 & 493 & 1958 \\
\hline
\end{tabular}

Los bosques de la RNT con mayor similitud fueron el Bosque de Tierra Firme y el Bosque Sucesional. Por otro lado, los más disímiles fueron el Bosque de Aguajal y el Bosque Sucesional. Tales diferencias se reflejan en el gráfico de la Figura 2 del NMDS con stress de Bray-Curtis $=0.08$ y varianza significativa $(p<0.001)$.

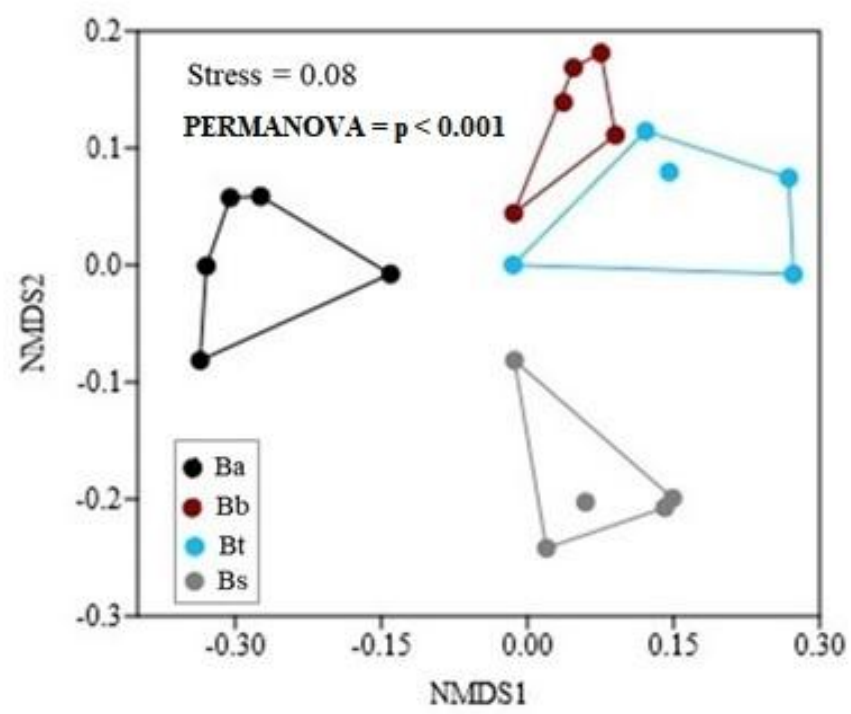

$\mathrm{Ba}=$ Bosque de Aguajal; $\mathrm{Bb}=$ Bosque de Bajío; $\mathrm{Bt}=$ Bosque de Tierra Firme;

$\mathrm{Bs}=$ Bosque Sucesional. Cada punto representa una parcela de $50 \times 30 \mathrm{~m}$ en la RNT, Madre de Dios.

Figura 2. NMDS de especies de los cuatro bosques en estudio.

Asimismo, el método de rarefacción-extrapolación, basado en las muestras mostró diferencia entre la riqueza específica y la heterogeneidad ambiental de las comunidades, el cual permitió constatar mayor riqueza en el Bosque de Tierra Firme y de Bajío (Figura 3). 


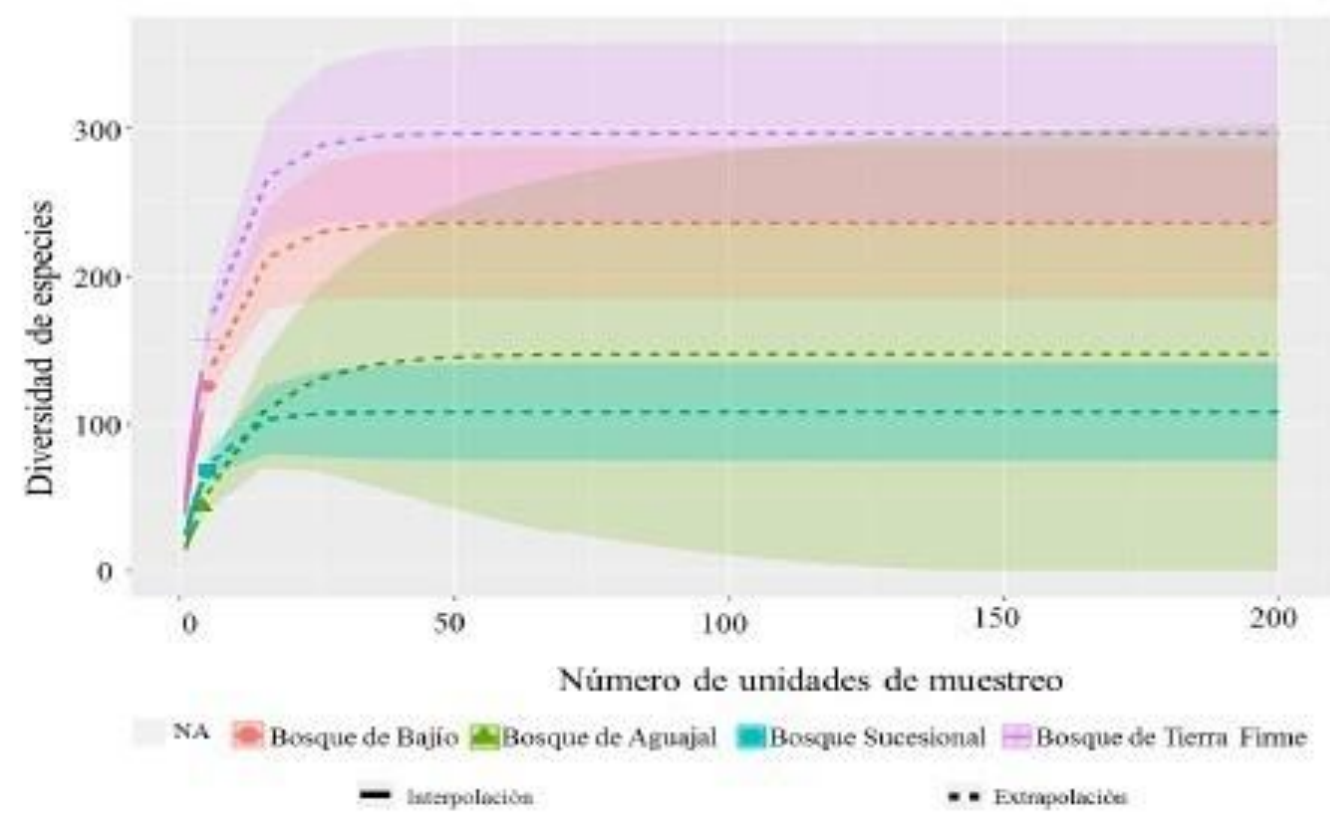

Figura 3. Curva de rarefacción de la riqueza estimada usando el índice de Chao2 para los bosques de la RNT, Madre de Dios.

\section{Análisis de especies indicadoras}

EI ISA identificó ocho especies como indicadoras para los respectivos bosques; las más destacadas: Mauritia flexuosa y Euterpe precatoria Mart., para el Bosque de Aguajal; Iriartea deltoidea Ruiz \& Pav., para el Bosque de Bajío; Erytrhina ulei, para el Bosque sucesional y Quararibea malacocalyx A. Robyns \& S. Nilsson, para el Bosque de Tierra Firme (Cuadro 3).

Cuadro 3. Especies indicadoras seleccionadas por ISA y utilizadas para el análisis $C C A$, de los bosques evaluados en la RNT.

\begin{tabular}{llcc}
\hline \multicolumn{1}{c}{ Tipo de Bosque } & \multicolumn{1}{c}{ Especie } & IndVal & p-valor \\
\hline \multirow{2}{*}{ Bosque de Aguajal } & Mauritia flexuosa L.f. & 1 & 0.005 \\
& Euterpe precatoria Mart. & 0.973 & 0.005 \\
\hline \multirow{2}{*}{ Bosque de Bajío } & Iriartea deltoidea Ruiz \& Pav. & 0.959 & 0.005 \\
& Otoba parvifolia (Markgr.) A.H. Gentry & 0.722 & 0.030 \\
\hline \multirow{2}{*}{ Bosque Sucesional } & Erythrina ulei Harms. & 0.946 & 0.005 \\
& Spondias mobin L. & 0.843 & 0.005 \\
\hline \multirow{2}{*}{ Bosque de Tierra Firme } & Quararibea malacocalyx A. Robyns \& S. Nilsson & 0.849 & 0.005 \\
& Tetragastis panamensis (Engl.) Kuntze & 0.775 & 0.030 \\
\hline
\end{tabular}


El análisis de $C C A$, basado en los registros de las fenofases de las especies indicadoras y en las variables ambientales de temperatura y precipitación demostró ser significativo $(M C=0.001)$; con la variable precipitación fuertemente correlacionada al eje 1 (-0.99); en cuanto a la variable temperatura al eje $2(-0.84)$. En el gráfico que tiene $22 \%$ de variación acumulada (Figura 4), se observa que ambas variables ambientales estuvieron muy correlacionadas con los meses de noviembre a marzo; precisamente, los meses con las mayores temperaturas y precipitaciones que corresponden al verano neotropical. Asimismo, las fenofases botón (2.65) y flor (2.55) presentaron los valores canónicos más altos para el eje 1 ; el fruto maduro $(-1.61)$ y fruto inmaduro (1.52) registraron mayores valores para el eje 2 (Figura 5).

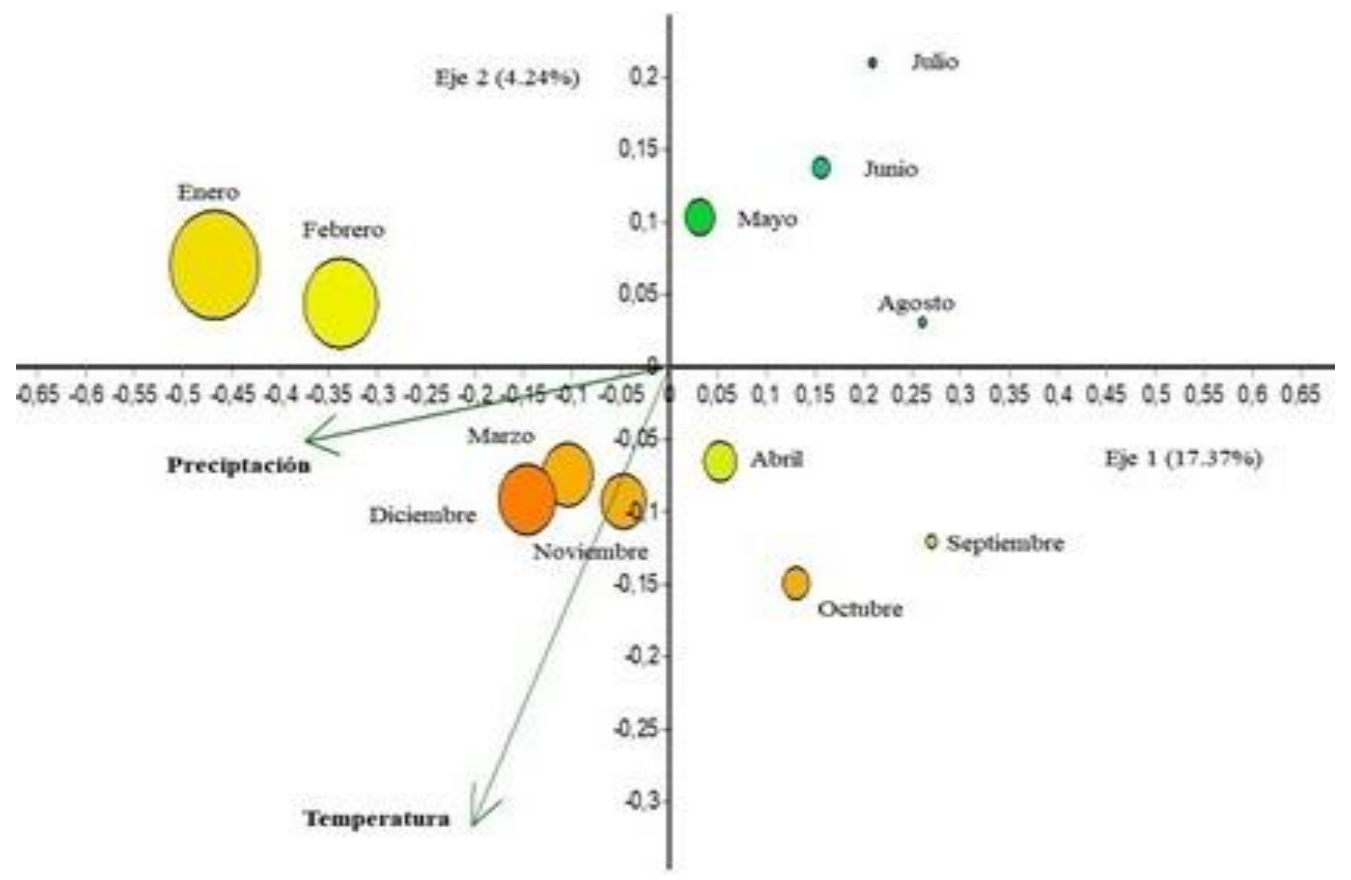

Círculos mayores $=$ Meses con mayor influencia de la precipitación; Escala de colores =Influencia de la variable temperatura, donde variaciones de azul y verde, meses con menores temperaturas; amarillo y naranja, meses con mayores temperaturas.

Figura 4. CCA de las variables ambientales en los bosques evaluados en la RNT, Madre de Dios. 


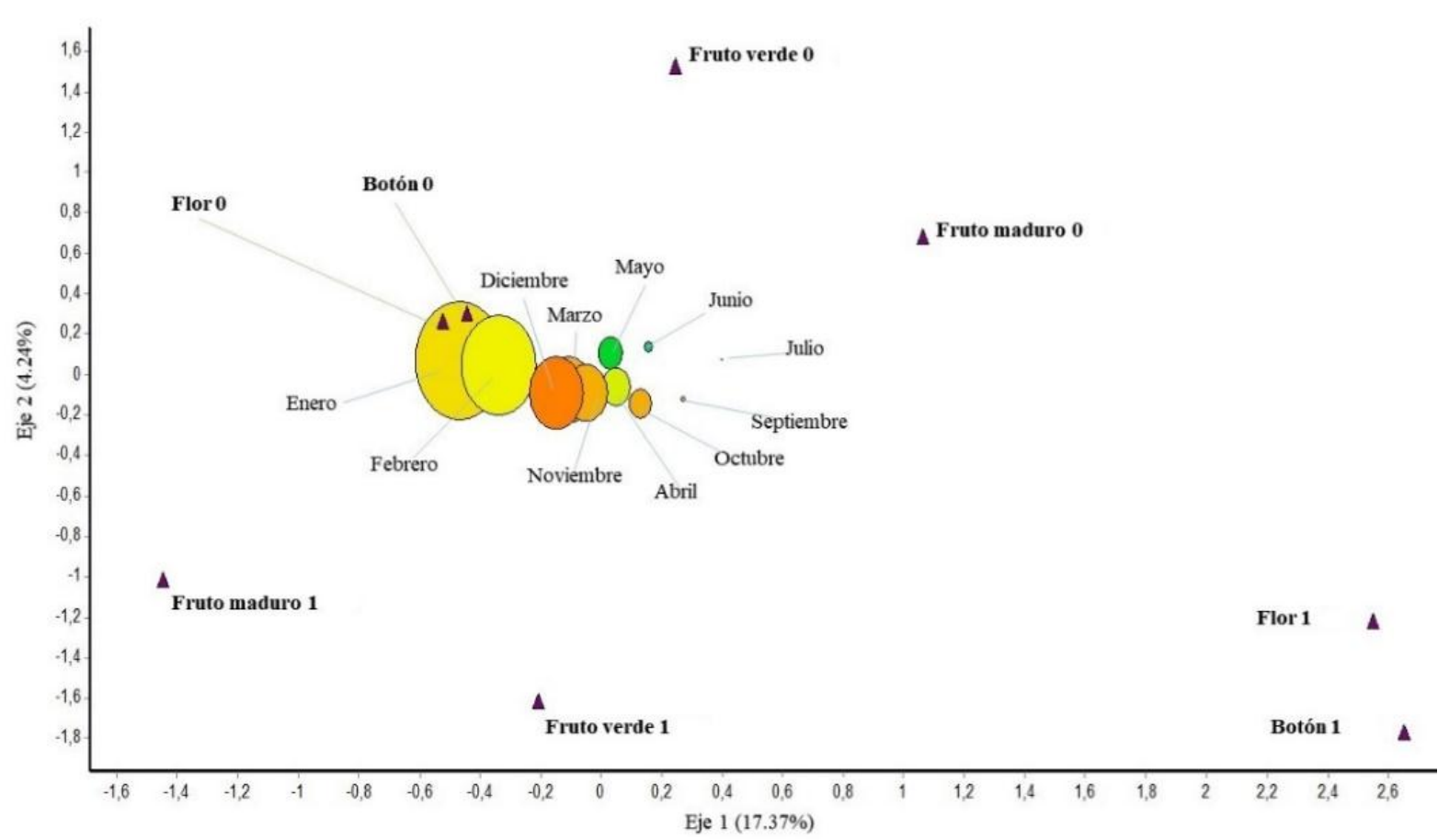

Círculos mayores = Meses con mayor influencia de la precipitación; Escala de colores = Influencia de la variable temperatura, donde variaciones de azul y verde, meses con menores temperaturas; amarillo y naranja, meses con mayores temperaturas.

Figura 5. CCA de las fenofases en la RNT, Madre de Dios.

El análisis de las fenofases de las especies indicadoras (Cuadro 4) evidenció que en el Bosque de Aguajal la fenofase botón floral se inició en octubre y alcanzó su máximo valor en noviembre (21\%), y los mayores valores de fruto maduro se alcanzaron en diciembre (16 \%) y enero (15\%). En el Bosque de Bajío el valor más alto de la fenofase botón floral (17\%) se obtuvo en septiembre y de fruto maduro en diciembre (29 \%). En el Bosque Sucesional el máximo valor de la fenofase botón floral (14\%) se registró en septiembre y de fruto maduro (23\%) en diciembre; en el Bosque de Tierra Firme el valor más alto de la fenofase botón floral se observó en septiembre (35\%), mientras que la de fruto maduro en enero (24\%). 
Cuadro 4. Registro de las fenofases de las especies indicadoras y media de variables ambientales en la RNT, Madre de Dios (2010-2017).

\begin{tabular}{|c|c|c|c|c|c|c|c|c|c|c|c|}
\hline \multirow{3}{*}{ Tipos de bosque } & \multicolumn{3}{|c|}{$\begin{array}{c}\text { Media mensual } \\
(2010-2017)\end{array}$} & \multicolumn{8}{|c|}{ Fenofases } \\
\hline & \multirow[b]{2}{*}{$\mathbf{M}$} & \multirow[b]{2}{*}{$\begin{array}{c}\mathrm{T} \\
\left({ }^{\circ} \mathrm{C}\right)\end{array}$} & \multirow[b]{2}{*}{$\begin{array}{c}\mathrm{Pp} \\
(\mathrm{mm})\end{array}$} & \multicolumn{2}{|c|}{ Botón } & \multicolumn{2}{|c|}{ Flor } & \multicolumn{2}{|c|}{$\begin{array}{c}\text { Fruto } \\
\text { inmaduro }\end{array}$} & \multicolumn{2}{|c|}{$\begin{array}{c}\text { Fruto } \\
\text { maduro }\end{array}$} \\
\hline & & & & $\begin{array}{c}0 \\
(\%)\end{array}$ & $\begin{array}{c}1 \\
(\%)\end{array}$ & $\begin{array}{c}0 \\
(\%)\end{array}$ & $\begin{array}{c}1 \\
(\%)\end{array}$ & $\begin{array}{c}0 \\
(\%)\end{array}$ & $\begin{array}{c}1 \\
(\%)\end{array}$ & $\begin{array}{c}0 \\
(\%)\end{array}$ & $\begin{array}{c}1 \\
(\%)\end{array}$ \\
\hline \multirow{12}{*}{ Bosque de Aguajal } & $E$ & 25.5 & 505.3 & 100 & 0.0 & 99.7 & 0.3 & 79.7 & 20.3 & 84.7 & 15.3 \\
\hline & $F$ & 25.4 & 434.0 & 99.8 & 0.2 & 99.9 & 0.1 & 83.3 & 16.7 & 87.1 & 12.9 \\
\hline & $M$ & 25.7 & 316.9 & 99.8 & 0.2 & 99.0 & 1.0 & 83.7 & 16.3 & 88.7 & 11.3 \\
\hline & $A$ & 25.3 & 224.3 & 98.8 & 1.2 & 96.4 & 3.6 & 81.5 & 18.5 & 96.1 & 3.9 \\
\hline & $M$ & 24.2 & 208.9 & 96.7 & 3.3 & 91.8 & 8.2 & 83.3 & 16.7 & 93.9 & 6.1 \\
\hline & J & 23.7 & 130.8 & 97.0 & 3.0 & 93.6 & 6.4 & 79.3 & 20.7 & 92.7 & 7.3 \\
\hline & $\mathrm{J}$ & 23.1 & 88.4 & 98.9 & 1.1 & 93.1 & 6.9 & 78.7 & 21.3 & 91.8 & 8.2 \\
\hline & $A$ & 24.2 & 87.3 & 99.1 & 0.9 & 91.8 & 8.2 & 76.9 & 23.1 & 88.9 & 11.1 \\
\hline & $\mathrm{S}$ & 25.2 & 105.8 & 99.8 & 0.2 & 98.7 & 1.3 & 76.4 & 23.6 & 88.2 & 11.8 \\
\hline & 0 & 25.7 & 192.5 & 87.0 & 13.0 & 99.6 & 0.4 & 81.4 & 18.6 & 87.9 & 12.1 \\
\hline & $\mathrm{N}$ & 25.7 & 286.4 & 78.9 & 21.1 & 87.2 & 12.8 & 80.5 & 19.5 & 85.8 & 14.2 \\
\hline & $\mathrm{D}$ & 25.9 & 344.3 & 99.4 & 0.6 & 95.1 & 4.9 & 76.0 & 24.0 & 84.3 & 15.7 \\
\hline \multirow{12}{*}{ Bosque de Bajío } & $E$ & 25.5 & 505.3 & 98.3 & 1.7 & 98.4 & 1.6 & 66.8 & 33.2 & 71.1 & 28.9 \\
\hline & $\mathrm{F}$ & 25.4 & 434.0 & 99.6 & 1.4 & 99.5 & 0.5 & 73.2 & 26.8 & 74.0 & 26.0 \\
\hline & $M$ & 25.7 & 316.9 & 99.4 & 1.6 & 99.6 & 0.4 & 72.0 & 28.0 & 75.4 & 24.6 \\
\hline & $A$ & 25.3 & 224.3 & 100 & 0.0 & 99.8 & 0.2 & 74.7 & 25.3 & 82.2 & 17.8 \\
\hline & $M$ & 24.2 & 208.9 & 96.9 & 3.1 & 99.0 & 1.0 & 76.7 & 23.3 & 84.3 & 15.7 \\
\hline & $\mathrm{J}$ & 23.7 & 130.8 & 96.1 & 3.9 & 95.7 & 4.3 & 78.8 & 21.2 & 79.8 & 20.2 \\
\hline & $\mathrm{J}$ & 23.1 & 88.4 & 90.9 & 9.1 & 92.4 & 7.6 & 85.7 & 14.3 & 80.5 & 18.5 \\
\hline & $A$ & 24.2 & 87.3 & 90.4 & 9.6 & 86.5 & 3.5 & 85.4 & 14.6 & 78.0 & 22.0 \\
\hline & $\mathrm{S}$ & 25.2 & 105.8 & 83.4 & 16.6 & 81.2 & 8.8 & 81.0 & 19.0 & 77.5 & 22.5 \\
\hline & 0 & 25.7 & 192.5 & 96.5 & 3.5 & 92.3 & 7.7 & 70.5 & 29.5 & 81.3 & 18.7 \\
\hline & $\mathrm{N}$ & 25.7 & 286.4 & 95.8 & 4.2 & 94.2 & 5.8 & 64.3 & 35.7 & 77.9 & 22.1 \\
\hline & $\mathrm{D}$ & 25.9 & 344.3 & 99.0 & 1.0 & 97.6 & 2.4 & 62.8 & 37.2 & 70.8 & 29.2 \\
\hline \multirow{11}{*}{ Bosque Sucesional } & $E$ & 25.5 & 505.3 & 99.5 & 0.5 & 100 & 0.0 & 83.4 & 16.6 & 79.6 & 20.4 \\
\hline & $F$ & 25.4 & 434.0 & 99.4 & 0.6 & 99.2 & 0.8 & 87.9 & 12.1 & 83.2 & 16.8 \\
\hline & $M$ & 25.7 & 316.9 & 99.0 & 1.0 & 99.6 & 0.4 & 89.3 & 10.7 & 90.7 & 9.3 \\
\hline & $A$ & 25.3 & 224.3 & 97.1 & 2.9 & 99.3 & 0.7 & 87.9 & 12.1 & 96.7 & 3.3 \\
\hline & $M$ & 24.2 & 208.9 & 91.0 & 9.0 & 99.3 & 0.7 & 84.0 & 16.0 & 98.4 & 1.6 \\
\hline & $\mathrm{J}$ & 23.7 & 130.8 & 92.0 & 8.0 & 99.0 & 0.1 & 79.9 & 20.1 & 99.0 & 1.0 \\
\hline & J & 23.1 & 88.4 & 90.8 & 9.2 & 98.0 & 2.0 & 83.0 & 17.0 & 95.4 & 4.6 \\
\hline & $A$ & 24.2 & 87.3 & 90.5 & 9.5 & 92.8 & 7.2 & 81.3 & 18.7 & 96.0 & 4.0 \\
\hline & $S$ & 25.2 & 105.8 & 86.0 & 14.0 & 96.1 & 3.9 & 78.2 & 21.8 & 93.1 & 6.9 \\
\hline & $\mathrm{O}$ & 25.7 & 192.5 & 95.8 & 4.2 & 90.0 & 10.0 & 67.2 & 32.8 & 91.7 & 8.3 \\
\hline & $\mathrm{N}$ & 25.7 & 286.4 & 99.0 & 1.0 & 98.6 & 1.4 & 67.0 & 33.0 & 83.0 & 17.0 \\
\hline
\end{tabular}




\begin{tabular}{cccccccccccc}
\hline & D & 25.9 & 344.3 & 99.4 & 0.6 & 100 & 0.0 & 76.0 & 24.0 & 77.2 & $\mathbf{2 2 . 8}$ \\
\hline & E & 25.5 & 505.3 & 99.7 & 0.3 & 100 & 0.0 & 81.9 & 18.1 & 76.0 & $\mathbf{2 4 . 0}$ \\
& F & 25.4 & 434.0 & 99.0 & 1.0 & 100 & 0.0 & 99.3 & 0.7 & 92.0 & 8.0 \\
& M & 25.7 & 316.9 & 92.2 & 7.8 & 96.8 & 3.2 & 100 & 0.0 & 98.2 & 1.8 \\
& A & 25.3 & 224.3 & 90.1 & 9.9 & 96.5 & 3.5 & 98.8 & 1.2 & 98.3 & 1.7 \\
& M & 24.2 & 208.9 & 87.8 & 12.2 & 93.0 & 7.0 & 96.5 & 3.5 & 100 & 0.0 \\
Bosque de Tierra Firme & J & 23.7 & 130.8 & 95.8 & 4.2 & 91.0 & 9.0 & 95.2 & 4.8 & 100 & 0.0 \\
& J & 23.1 & 88.4 & 99.1 & 0.9 & 99.0 & 1.0 & 93.6 & 6.4 & 98.6 & 1.4 \\
& A & 24.2 & 87.3 & 90.5 & 9.5 & 97.7 & 2.3 & 93.6 & 6.4 & 99.1 & 0.9 \\
& S & 25.2 & 105.8 & 65.5 & $\mathbf{3 4 . 5}$ & 74.1 & $\mathbf{2 5 . 9}$ & 95.0 & 5.0 & 96.8 & 3.2 \\
& O & 25.7 & 192.5 & 94.5 & 5.5 & 95.6 & 4.4 & 60.1 & $\mathbf{3 9 . 9}$ & 96.3 & 3.7 \\
& N & 25.7 & 286.4 & 99.7 & 0.3 & 99.7 & 0.3 & 62.0 & $\mathbf{3 8 . 0}$ & 92.0 & 8.0 \\
& D & 25.9 & 344.3 & 99.0 & 1.0 & 99.5 & 0.5 & 70.9 & 29.1 & 83.9 & $\mathbf{1 6 . 1}$ \\
\hline
\end{tabular}

\section{Discusión Composición florística}

Es importante conocer las dimensiones de la heterogeneidad florística para describir y comprender los patrones dinámicos de las especies y su interacción con el ambiente. El presente estudio evidencia que las especies indicadoras analizadas con frecuencia se citan en estudios florísticos locales (Baez y Garate, 2017; Dueñas y Garate, 2018; Alvarez-Montalván et al., 2021). Las familias, como Fabaceae, Moraceae y Arecaceae se consignan como representativas de los bosques amazónicos del Perú (Vásquez et al., 2010; Ureta, 2015, Alvarez-Montalván et al., 2021).

De la misma manera, el agrupamiento específico de individuos por tipo de bosque permite establecer similitudes cercanas entre hábitats. Es así que el análisis de agrupamiento NMDS evidenció que hábitats con mayores números de individuos arbóreos, como el Bosque de Bajío y Bosque de Tierra Firme, presentaban más similitud que los bosques con menor cantidad de individuos. Por otro lado, se ha indicado que el análisis tiene la desventaja de generar más de una respuesta, por lo que resulta necesario llevar a cabo procedimientos para determinar el menor valor de tensión (Rocha-Loredo et al., 2010). En ese 
sentido, la confiabilidad del análisis realizado válido; puesto que, el valor de stress estuvo por debajo de 0.1 .

Adicionalmente, la comparación directa de la riqueza observada por tipo de hábitats mostró cómo habitas no correlacionados por el análisis de agrupamiento presentaron más especies, como el caso de los Bosque de Bajío y el Bosque Sucesional. Sin embargo, ninguno de los tipos de bosques alcanzó la estabilidad en el número de taxones indicada por el coeficiente indicador de Chao2. En complemento, Samaniego et al. (2015) con el mismo análisis de rarefacción, no obtuvieron diferencias en la riqueza. En contraste, Silva et al. (2016) indicaron que la estabilidad de la curva de rarefacción en el Bosque Nacional Tapajós se observó, especialmente, para los bosques secundarios.

\section{Análisis de las respuestas fenológicas de las especies indicadoras}

Las relaciones entre los diferentes tipos de hábitats y los parámetros meteorológicos evidenciaron mayores variaciones de las respuestas fenológicas en el Bosque de Tierra Firme, lo que indicaría más sensibilidad a los cambios de temperatura y precipitación. En cambio, al correlacionar los parámetros ambientales en estudio para los Bosques de Aguajal, de Bajío y Sucesional estos serían influenciados más por la temperatura que la precipitación. La ausencia de precipitaciones o sequías extremas y la temperatura, dependiendo de los periodos de duración (Siegmund et al., 2016), afectarían a los individuos arbóreos del Bosque de Tierra Firme que se encuentran en la Amazonía (Zhao et al., 2017). Uno de los indicadores inmediatos es la pérdida de verdor de los bosques y la senescencia de hojas; lo que conlleva a la acumulación de material orgánico, descomposición y aumento de $\mathrm{CO}_{2}$. Al respecto, en el estudio de Pires et al. (2018), realizado en una reserva de Mata Atlántica de Río de Janeiro, se determinó una relación débil entre las condiciones climáticas y la dinámica de formación y maduración de frutos, la que se relacionó con la fenodinámica de la floración. Por el contrario, en el presente estudio, se observó que la presencia de frutos inmaduros y maduros disminuyó, de manera ostensible, conforme se 
aproximaban los meses de junio a septiembre, época en que la precipitación alcanzó sus menores valores.

El comportamiento fenológico de las plantas, como indicador del cambio climático, está siendo investigado y monitoreado en diversos lugares del mundo (Workie y Debella, 2018). En estos estudios el comportamiento fenológico resultará determinante en el análisis e interpretación de los componentes climáticos en el tiempo y el espacio (Pires et al., 2018), tal como se observa en el estudio que aquí se documenta. Es por ello que, al margen de otros factores ambientales no considerados, el efecto de la temperatura y precipitación resultaron significativos en el establecimiento de las fenofases de las especies evaluadas.

Por otra parte, los bosques tropicales se caracterizan por presentar una fenología con alta diversidad debido a que carecen de una estación fría bien definida que afecte y restringa su crecimiento. Por esto, es posible observar especies con flores y frutos todo el año, otras que florecen y fructifican una vez al año, o una sola vez en varios años (Pires et al., 2018). De igual forma, la mayoría de las plantas leñosas tropicales producen nuevas hojas y flores de manera repentina y no continuamente, por lo que sus cambios fenológicos representan adaptaciones a factores bióticos y abióticos (Van Schaik et al., 2003).

Es importante destacar que, aunque la contribución de los resultados del presente estudio es limitada, constituye una base de apoyo al conocimiento científico local, y refleja la necesidad de integrar mayores números de especies, con el fin de no discriminar posibles taxones que podrían ser fundamentales para el entendimiento de la fenodinámica del área de estudio. Por lo tanto, es imperativo la conservación de la reserva y sus hábitats. 


\section{Conclusiones}

Los resultados muestran que las especies indicadoras constatadas en este estudio, además de representar las familias más abundantes de la amazonia peruana demostraron que sus fenofases están correlacionadas con la temperatura y la precipitación. Sin embargo, proporcionan apenas un patrón inicial de la fenología de las especies, por lo que se recomienda la continuidad de investigaciones en esa línea, con el fin de llenar lagunas científicas y contribuir con la conservación del área.

\section{Agradecimientos}

A la Sociedad Pro Guacamayos, The Macaw Society, en la persona del Dr. Donald J. Brightsmith.

\section{Conflicto de interés}

Los autores declaran no tener conflicto de intereses.

\section{Contribución de los autores}

Gustavo A. Martínez-Sovero: planeación del proyecto, toma de datos de campo y revisión del manuscrito; Consuelo Rojas-Idrogo, planeación y supervisión del proyecto y revisión del manuscrito; Guillermo E. Delgado-Paredes, redacción y revisión del manuscrito; Felipe Zuñe-Da Silva, análisis de datos; Alexander Huamán-Mera, redacción y revisión del manuscrito; Yuriko S. Murillo-Domen, redacción y revisión del manuscrito; Donald J. Brightsmith, redacción y revisión del manuscrito. Todos los miembros del equipo aprobaron el manuscrito final. 


\section{Referencias}

Angiosperm Phylogeny Group (APG IV). 2016. An update of the Angiosperm Phylogeny Group classification for the orders and families of flowering plants: APG IV. Botanical Journal of the Linnean Society 181:1-20. Doi:10.1111/boj.12385.

Alvarez-Montalván, C. E., Manrique-León, S., Vela-Da Fonseca, M., CardozaSoarez, J., Callo-Ccorcca, J., Bravo-Camara, P., Castañeda-Tinco, I. and AlvarezOrellana, J. 2021. Floristic composition, structure and tree diversity of an Amazon forest in Peru. Scientia Agropecuaria 12(1): 73-82. Doi:10.17268/sci.agropecu.2021.009.

Baez, Q. S. M. y J. S. Garate Q. 2017. Estructura y composición florística arbórea en dos tipos de bosque en la Zona de Amortiguamiento, Reserva Nacional de Tambopata. Q'EUÑA 8(1): 39-50. DOI:10.51343/rq.v8i1.103.

Colwell, R. K. 2013. EstimateS: Statistical estimation of species richness and shared species from samples. Version 9. User's Guide and application. Doi: 10.1613/jair.301.

Daru, B. H., M. M. Kling, E. K. Meineke and A. E. van Wyk. 2019. Temperature controls phenology in continuously flowering Protea species of subtropical Africa. Applications in Plant Sciences 7(3):e1232. Doi: 10.1002/aps3.1232.

Davies, T. J., E. M. Wolkovich, N. J. B. Kraft, N. Salamin, J. M. Allen, T. R. Ault, J. L. Betancourt, K. Bolmgren, E. E. Cleland, T. M. Crimmins, S. J. Mazer, G. J. McCabe, S. Pau, J. Regetz, M. D. Schwartz and S. E. Travers. 2013. Phylogenetic conservatism in plant phenology. Journal of Ecology 101:1520-1530. Doi:10.1111/1365-2745.12154.

Du, Y., L. Mao, S. A. Queenborough, R. P. Freckleton, B. Chen and K. Ma. 2015. Phylogenetic constraints and trait correlates of flowering phenology in the angiosperm flora of China. Global Ecology and Biogeography 24:928-938. Doi:10.1111/geb.12303. 
Dueñas, L. H. y J. S. Garate. 2018. Diversidad, dominancia y distribución arbórea en Madre de Dios, Perú. Revista Forestal del Perú 33(1): 4-23. Doi: $10.21704 /$ rfp.v33i1.1152.

Dufrêne, M. and P. Legendre. 1997. Species assemblages and indicator species: the need for a flexible asymmetrical approach. Ecological Monographs 67:345366. Doi:10.2307/2963459.

Fournier, L. A. 1974. Un método cuantitativo para la medición de características fenológicas en árboles. Turrialba 24:422-423. http://orton.catie.ac.cr/repdoc/A077e/A0773e04.html (15 de octubre de 2021).

Girardin, C. A. J., Y. Malhi, C. E. Doughty, D. B. Metcalfe, P. Meir, J. del AguilaPasquel, A. Araujo-Murakami, A. C. L. da Costa, J. E. Silva-Espejo, F. Farfán A. and L. Rowlands. 2016. Seasonal trends of Amazonian rainforest phenology, net productivity, and carbon allocation. Global Biogeochemical Cycles 30:700-715. Doi: $10.1002 / 2015 G B 005270$.

Häder, D.-P. and P. W. Barnes. 2019. Comparing the impacts of climate change on the responses and linkages between terrestrial and aquatic ecosystems. Science of the Total Environment 682:239-246. Doi: 10.1016/j.scitotenv.2019.05.024.

Jackson, S. D. 2009. Plant responses to photoperiod. New Phytologist 181:517531. Doi: 10.1111/j.1469-8137.2008.02681.x.

Körner, C. and D. Basler. 2010. Phenology under global warming. Science 327:1461-1462. Doi:10.1126/science.1186473.

Kruskal, J. B. 1964. nonMetric Multidimensional scaling - a numerical method. Psychometrika 29:115-129. http://cda.psych.uiuc.edu/psychometrika_highly_cited_articles/kruskal_1964b.pd $f$ (15 de octubre de 2021).

Matsumoto, K., T. Ohta, M. Irasawa and T. Nakamura. 2003. Climate change and extensión of the Ginkgo biloba L. growing season in Japan. Global Change Biology 9:1634-1642. Doi: 10.1046/j.1365-2486.2003.00688.x. 
McCune, B. and J. B. Grace. 2002. Analysis of Ecological Communities. Journal of Experimental Marine Biology and Ecology. Vol. 289. MJM Software Design. Doi:10.1016/S0022-0981(03)00091-1.

Pires, J. P. A., N. A. C. Marino, A. G. Silva, P. J. F. P. Rodrigues and L. Freitas. 2018. Tree community phenodynamics and its relationship with climatic conditions in a lowland tropical rainforest. Forests 9:114. Doi: 10.3390/f9030114.

Phillips, O., T. Baker, T. Feldpausch and R. Brienen. 2016. Manual de campo para el establecimiento y la remedición de parcelas. RAINFOR, The Royal Society. Londres, Inglaterra. $28 \mathrm{p}$.

R Core Team. 2021. A Language and Environment for Statistical Computing. R Foundation for Statistical Computing. Austria, Vienna. 105 p.

Ribeiro, J. E. L. S., M. J. G. Hopkins, A. Vicentini, C. A. Sothers, M. A. S. Costa, J. M. Brito, M. A. D. Souza, L. H. Martins, L. G. Lohmann, P. A. Assunção, E. C. Pereira, C. F. Silva, M. R. Mesquita e L. C. Procópio. 1999. Flora da Reserva Ducke. Guia de identificação das plantas vasculares de uma floresta de terra firme na Amazônia Central. INPA-DFID. Manaus, Brasil. 800 p. Doi: $10.2307 / 4110841$.

Rocha-Loredo, A. G., N. Ramírez-Marcial y M. González-Espinosa. 2010. Riqueza y diversidad de árboles del bosque tropical caducifolio en la Depresión Central de Chiapas. Boletín de la Sociedad Botánica de México 87:89-103. Doi.org/10.17129/botsci.313.

Samaniego, E., Y. García, D. Neill, Y. Arteaga, J. C. Vargas y L. Rojas. 2015. Diversidad florística de tres sitios de un bosque siempreverde piemontano de la región oriental amazónica del Ecuador. Revista Amazónica Ciencia y Tecnología 4:32-47. https://dialnet.unirioja.es/servlet/articulo?codigo $=5271973 \quad$ (5 de octubre de 2021).

Shepherd, G. J. 2010. Fitopac 2.1. Departamento de Botânica/UNICAMP. São Paulo, Brasil. http://m.pedroeisenlohr.webnode.com.br/fitopac/ (15 de octubre de 2021). 
Siegmund, J., M. Wiedermann, J. Donges and R. Donner. 2016. Impact of temperatura and precipitation extremes on the flowering date of four German widlife shrub species. Biogeosciences 13:5541-5555. Doi:10.51914/bg-5541-2016.

Silva, C. V., J. R. dos Santos, L. S. Galvão, R. D. da Silva and Y.M. Moura. 2016. Floristic and structure of an Amazonian primary forest and a chronosequence of secondary succession. Acta Amazonica 46:133-150. Doi: 10.1590/18094392201504341.

Ureta, M. 2015. Aporte de biomasa aérea de las especies arbóreas de la familia Myristicaceae en los bosques amazónicos del Perú. Revista Biología Tropical (International Journal of Tropical Biology) 63:263-273. Doi:10.15517/RBT.V63I1.14254.

Van Schaik, C. P., J. Terborgh and W. S. Joseph. 2003. The phenology of tropical forests: Adaptative significance and consequences for primary consumers. Annual Review of Ecology and Systematics 24:353-377. Doi:10.1146/annurev.es.24.110193.002033.

Vásquez, R., R. Rojas y H. Van der Werff. 2010. Flora del Río Cenepa, Amazonas, Perú. Missouri Botanical Garden. St. Louis, MO, USA. 1557 p.

Wagner, F. H., Hérault, B., Rossi, V., Hilker, T., Maeda, E. E., Sanchez, A., Lyapustin, A. I., Galvão, L. S., Wang, Y. and Aragão, L. E. O. C. 2017. Climate drivers of the Amazon forest greening. PLos ONE 12(7): e0180932. Doi: $10.1371 /$ journal. pone.0180932.

Wolkovich, E. M., B. I. Cook, J. M. Allen, T. M. Crimmins, J. L. Betancourt, S. E. Travers, S. Pau, J. Regetz, T. J. Davies, N. J. B. Kraft, T. R. Ault, K. Bolgren, S. J. Mazer, G. J. McCabe, B. J. McGill, C. Parmesan, N. Salamin, M. D. Schawartz and E. E. Cleland. 2012. Warming experiments underpredict plant phenological responses to climate change. Nature 485:494-497. Doi: 10.1038/nature11014.

Workie, T. G. and H. J. Debella. 2018. Climate change and its effects on vegetative phenology across ecoregions of Ethiopia. Global Ecology and Conservation 13:e00366. Doi:10.1016/j.gecco.2017.e00366. 
Zhao, W., Z. Zhao, T. Zhou, D. Wu, B. Tang B and H. Wei. 2017. Climate factors driving vegetation declines in the 2005 and 2010 Amazon droughts. PLoS ONE 12(4):e0175379. Doi:10.1371/journal.pone.0175379.

\section{(c) (7) (9)}

Todos los textos publicados por la Revista Mexicana de Ciencias Forestales -sin excepción- se distribuyen amparados bajo la licencia Creative Commons 4.0 Atribución-No Comercial (CC BY-NC 4.0 Internacional), que permite a terceros utilizar lo publicado siempre que mencionen la autoría del trabajo y a la primera publicación en esta revista. 